\title{
ABANDONO Y DISCONTINUIDAD \\ DEL TRATAMIENTO \\ DE INFERTILIDAD
}

Cristina Di Silvestre

\section{RESUMEN:}

Un grupo de 50 mujeres infértiles que abandonaron y/o discontinuaron el estudio y tratamiento de su infertilidad fueron entrevistadas con el fin de conocer las causas y motivos de abandono, como los factores asociados a aquel fenómeno. Se descubrió que la mujer inicia el estudio y tratamiento con escaso o ningún conocimiento y sus expectativas en relación al costo, duración y tipo de exámenes están distante de la realidad que enfrenta al respecto una vez iniciado el tratamiento. Presenta un bajo nivel de tolerancia a los exámenes y reconoce haber tenido trastornos fisiológicos y cambios en el estado afectivo después de iniciado el estudio de su infertilidad. Los problemas que enfrenta la mujer en la realización de los exámenes son: trabajo, discusiones con su pareja y dinero.

Los motivos de abandono más frecuentemente mencionados se refiere a la "relación médico-paciente" $(50 \%)$, en una proporción menor $(20 \%)$ se aludió a motivos relacionados con "los efectos del tratamiento", similar porcentaje a motivos de "la relación de pareja" y un 10\% los "económicos". El estudio sugiere la existencia de una insatisfacción de las expectativas de la mujer vinculadas al componente expresivo (aspectos humanos y afectivos) de la relación médico-paciente que estaría contribuyendo a la decisión de abandono y o discontinuación del tratamiento.

\section{INTRODUCCIÓN}

No existen estudios dentro de nuestro medio acerca de las causas, motivos, incidencia y frecuencia del abandono y discontinuidad del tratamiento de la infertili- 
dad. Estimaciones realizadas en algunos Servicios Públicos de Salud de Santiago ponen en evidencia que la tasa de abandono fluctúa entre un $20 \%$ y un $70 \%$. Esta diferencia tan acentuada entre el porcentaje mínimo y máximo deja un interrogante acerca de qué factores actúan reteniendo a la pareja en el tratamiento, los cuales al estar ausentes, estarían aumentando el riesgo de abandono y discontinuidad. No sólo es la pareja la que se perjudica al abandonar o discontinuar el tratamiento, dado por un lado que su problema de infertilidad continúa y el costo psicológico que éste implica (temor de esterilidad definitiva, pérdida de la intimidad sexual, etc.) sino por otro lado también los servicios médicos toda vez que deben destinar un tiempo prolongado y recursos importantes, tanto materiales y humanos, en la atención de una sola pareja. La alta tasa de abandono también perjudica a aquellas parejas que no asisten a tratamiento: se percibe el tratamiento como con pocas posibilidades de éxito, como muy difícil y por tanto no asisten a solicitarlo. Numerosas y de diversas indole pueden ser las variables que de una u otra manera pueden llevar a una pareja a abandonar o discontinuar el tratamiento de su infertilidad. Algunas parejas no desean continuar porque quieren evitar la vergüenza y el ridículo si se llega a descubrir que el factor responsable es el masculino (9). La sola suposición de que el varón puede ser el causante podría generar algún tipo de disfunción sexual y en particular las exigencias médicas en torno a la actividad sexual pueden desencadenar una impotencia temporal que limita y disminuye las probabilidades de éxito del tratamiento y aumenta el riesgo de abandono $\langle 21,22$, 1).

Desde un punto de vista psicológico varios podrían ser los motivos de abandono; el miedo a un aborto o a tener un hijo con malformaciones, de modo que la infertilidad puede actuar como mecanismo protector frente a la ansiedad generada por el miedo (17).

Aun cuando puede parecer paradójica, no siempre la asistencia a un tratamiento por infertilidad puede considerarse como evidencia suficiente de una motivación por tener un hijo; los médicos denominan "infertilidad psicógena" o "funcional" cuando una mujer dice desear un hijo, pero inconscientemente rechaza el embara$z o$, el parto y la maternidad $(3,6)$. Al respecto debemos mencionar el estigma y presión social que recibe la mujer cuando es estéril, siendo fértil declara no desear tener hijos $(3,21)$; un tratamiento prolongado puede servirle de justificación al hecho de no continuar asistiendo a consulta.

La crisis de la falta de hijos puede afectar de maneras distintas a cada pareja y a cada uno de sus miembros, desencadenando a veces problemas de salud: drogadicción, alcoholismo, anorexia u obesidad. Cuando esto ocurre la pareja abandona el tratamiento hasta tanto no se reestablezca la salud del miembro que está enfermo (15).

La edad de la mujer puede considerarse como una variable que podría llevar a la pareja a tomar la decisión de abandonar el tratamiento; la pareja sabe que si no 
hay embarazo en los primeros años de estudio de su infertilidad, las posibilidades van disminuyendo con el correr del tiempo $(8,10,14)$.

Un tiempo prolongado de tratamiento sin un resultado exitoso puede también llevar a la pareja a tomar la decisión de adoptar un niño y abandonar el tratamiento. El tiempo prolongado que se requiere para la implementación del tratamiento supone que la pareja disponga de tiempo, ciertas horas y días, lo que exige perseverancia y paciencia. Considerando que no siempre los resultados son concluyentes, la frustración sistemática al generar desesperanza, puede aumentar las probabilidades de abandono y discontinuidad del tratamiento.

Atendiendo a los planteamientos anteriores nos parece de crucial importancia realizar un estudio exploratorio del fenómeno del abandono y discontinuación del tratamiento de la infertilidad, de manera que una vez determinadas las causas y motivos más frecuentes se elabore un instrumento que permita predecir, en algún grado, el riesgo de abandono, para actuar de manera preventiva sobre las parejas de mayor riesgo.

\section{MATERIAL Y MÉTODO}

1. El grupo estudiado: estuvo constituido por 50 mujeres infértiles: 25 atendidas en Consulta Privada y 25 en Servicios Públicos de Salud. Para constituir ambos grupos se procedió a:

1.a) Solicitar las direcciones de pacientes que dejaron de asistir a tratamiento de infertilidad a lo menos seis meses. La solicitud se realizó en tres Consultas Privadas y dos Hospitales. Este procedimiento no fue todo lo exitoso que se esperaba; algunas direcciones eran falsas o algunas pacientes ya no vivían en la dirección registrada. Luego fue necesario complementar este procedimiento con el siguiente:

1.b)Contactar mujeres que actualmente se encuentran en tratamiento y que asistieron a un estudio de su infertilidad con otro especialista. Este contacto se hizo en los mismos Servicios de Salud y Consultas Privadas en los horarios de atención médica.

A pesar que los casos fueron reclutados en dos Hospitales y tres Consultas Privadas lo que podría hacer pensar que los datos recogidos carecen de representatividad y que no es posible generalizar las conclusiones, pudimos constatar que las mujeres asistieron a estudio de su infertilidad con distintos especialistas y Servicios de Salud y por ende ningún Hospital ni especialista específico constituye una selección intencionada, sino al azar.

Los criterios de selección de las mujeres infértiles que constituyeron el grupo para ser estudiado fueron: 1. Haber abandonado o discontinuado a lo menos durante seis meses el tratamiento antes de reiniciarlo, 2. Mujeres que nunca han 
tenido hijos. Ninguna mujer que tuvo hijos y que actualmente es infértil fue considerada para estudio.

Creemos que el total de 50 casos es una cifra aceptable para la fase exploratoria del estudio.

2. Instrumento de medición: la recolección de los datos se realizó mediante una cédula de entrevista que fue aplicada a algunas mujeres en sus hogares, a otras en la misma Consulta u Hospital y a otras en su lugar de trabajo, dependiendo del lugar que más le acomodara a la entrevistada.

La duración de la entrevista fue de una hora y quince minutos aproximadamente. Esto se debió a que en ella se abordaron varios aspectos de la situación de la mujer infértil dado el carácter exploratorio del estudio.

El trabajo de campo fue realizado entre los meses de octubre y diciembre de 1987, enero y marzo de 1988.

\section{RESULTADOS}

\section{Aspectos sociodemográficos de la mujer infértil}

La mayor parte de las mujeres de nuestro estudio son relativamente jóvenes; el $76 \%$ se encuentra entre 20 y 34 años. Sólo casi una cuarta parte del grupo tiene 35 años o más.

El Nivel Educacional de la mujer sigue la misma tendencia que la de su esposo; existe más cantidad de casos en las categorías de Enseñanza Superior $(46 \%$ y $42 \%$, esposos y esposas, respectivamente) que en la categoría de Enseñanza Básica ( $20 \%$ y $18 \%$, respectivamente). El número de mujeres es mayor $(40 \%)$ al de esposos (34\%) en el Nivel de Enseñanza Media.

Del total de mujeres entrevistadas, el $60 \%$ realiza una actividad laboral fuera de su hogar y el $40 \%$ es dueña de casa. Un poco menos de un tercio (30\%) pertenece al Estrato Socioeconómico Alto, un $18 \%$ al Estrato Medio y un $52 \%$ al Estrato Bajo.

Las dos quintas partes de las mujeres estudiadas tienen entre 4 y 6 años de matrimonio. Menos de un tercio (30\%) entre 7 y 10 años. Un $16 \%$ entre 1 y 3 años y un $14 \%$ entre 11 y 13 años.

\section{Acceso al tratamiento}

Más de la mitad del grupo estudiado fue aconsejada por un médico o una matrona para que recurriera a un especialista en infertilidad. Las amigas y parientes fueron los medios de contacto en un poco más de una cuarta parte del grupo $(26 \%)$. Un $14 \%$ declaró que se comunicó con el especialista por iniciativa propia, un $4 \%$ recibió la información en el Servicio de Seguro Social y un $2 \%$ mediante los medios de comunicación social.

Un poco más de un tercio (36\%) estuvo en estudio de su infertilidad con otro 
especialista. De estas mujeres los dos tercios esperó entre uno y tres meses antes de consultar a un nuevo especialista y el tercio restante reinició tratamiento después del tercer mes.

Del total de mujeres, sólo un poco menos de un tercio $(30 \%)$ asistía discontinuadamente a estudio. Estas mujeres aludieron a diferentes razones como explicación de su irregular asistencia: $40 \%$ declaró que el estudio les "aburría" y "cansaba", un $20 \%$ que estaban "desinformadas en cuanto a las fechas en que debían hacerse los exámenes", $26 \%$ por vacaciones y $13 \%$ por problemas de trabajo.

La mayoria de las mujeres alcanzó a estar un año en tratamiento (64\%) y menos de un quinto llega al año y medio y dos años y el $14 \%$ restante permanece más de dos años.

Del total de mujeres entrevistadas, un $58 \%$ se encuentra actualmente en estudio de su infertilidad. Del grupo restante ( 21 casos) sólo un $9 \%$ dijo que no reiniciaría el tratamiento por lo avanzado de su edad.

\section{Expectativas en relación al tratamiento}

Se estudiaron tres variables para determinar las expectativas de la mujer en relación al tratamiento: costo, duración y exámenes que el especialista le solicitaría. Se constató que la mitad del grupo esperaba que el tratamiento sería por poco tiempo, un $22 \%$ no sabía ni imaginaba cómo sería éste.

Más de la mitad del grupo ignoraba qué exámenes le pediría el médico (52\%), un $26 \%$ suponía que sería de "orina y sangre", la ecografía fue mencionada en un $10 \%$ de los casos y similar porcentaje se refirió al espermiograma. Sólo un $2 \%$ dijo saberlo todo. Dos quintas partes del grupo esperaba que el costo fuese "más bajo", para un porcentaje levemente menor (38\%) el costo fue similar a lo que esperaba y sólo un $6 \%$ supuso que sería "más alto". A un $14 \%$ no le preocupó el costo, unas porque les salió gratis y a otras porque disponian de dinero para pagarlo y no se plantearon expectativas al respecto.

\section{Tolerancia a los procedimientos solicitados por el especialista}

Con el propósito de conocer la tolerancia de la mujer a los exámenes que debió realizar incluimos algunas preguntas sobre reacciones antes, durante y después de los exámenes. Con respecto a cómo se sentía "antes" de hacerse la radiografía a las trompas, las tres quintas partes $(60 \%)$ dijo que estaba "intranquila y nerviosa", menos de un tercio (31\%) se sintió "tranquila" y un $9 \%$ "temerosa por los resultados". Dos de cada diez mujeres dijo haber sentido "dolor" durante el examen. A un poco más de la tres cuartas partes (36 casos) del total de mujeres, el médico le solicitó la realización de una Biopsia de Endometrio. De éstas, el $89 \%$ experimentó 
dolor durante el examen. Menor fue la proporción (62\%) de aquellas que también sintieron dolor "después".

A la mitad del grupo ( 25 casos) el médico le recetó un determinado medicamento. A menos de la mitad de este grupo le preocupó los efectos que pudiese tener en su organismo. El resto no tuvo preocupación al respecto.

\section{Problemas que se le presentan a la mujer infértil en la realización de los exámenes}

Entre las mujeres que trabajan fuera de su hogar, el trabajo es la mayor limitante para la realización de los exámenes. Del total de mujeres (tanto las que trabajan fuera del hogar como las que no lo hacen) casi las dos quintas partes hicieron mención al dinero y algunas $(24 \%)$ reconocieron haber tenido discusiones con la pareja.

\section{Nivel de conocimientos sobre infertilidad $y$ tratamiento de la infertilidad}

Con el propósito de obtener información sobre el Nivel de Conocimientos de que dispone la mujer que participa en un estudio de infertilidad se les preguntó si conocía las razones de que los exámenes no fueran realizados simultáneamente y si sabía cuáles eran los factores que podían impedir el embarazo en la mujer como aquellos que limitaban la fertilidad masculina.

La mitad del grupo aludió al "ciclo", éste iría determinando la oportunidad apropiada para la realización de los exámenes. Un porcentaje menor $(23 \%)$ de aquellas que aludieron a alguna razón ( 36 mujeres) dijo que dado que los exámenes implicaban mucha exigencia física de parte de la mujer debía dejarse un tiempo adecuado para que se restableciera. Un $14 \%$ que se iba haciendo "según avance del tratamiento". Un porcentaje menor $(11 \%)$ declaró que los exámenes debían ser realizados en condiciones diferentes y sólo un $2 \%$ dijo que la existencia de "más pacientes" impedía que a una sola se le hicieran todos los exámenes de una vez.

Al clasificar las respuestas sobre los factores que impiden el embarazo tenemos lo siguiente:

\section{FACTORES QUE IMPIDEN}

\section{EL EMBARAZO EN LA MUJER}

Problemas en aparato reproductor

Deficiencia en el funcionamiento hormonal

Factor Psicológico (estrés, nervios, etc.)

Presión Social

"Dios no quiere"

Abortos 
Carencia y/o dificultad en motilidad de los espermios Aparato genital dañado por enfermedad o accidente

Con respecto a la información que la mujer tenía ANTES de iniciar el tratamiento, más de la mitad (62\%) carecía de antecedentes. De las mujeres restantes ( 19 casos), casi un tercio sabía que "eran prolongados". Un poco más de un cuarto dijo saber que "eran efectivos", un $16 \%$ conocía las "posibles causas", similar porcentaje dijo que sabía que "se estudia a la mujer" y un $11 \%$ que eran "dolorosos".

\section{Información que le hubiese gustado recibir} ANTES Y DURANTE el tratamiento

Los dos tercios del grupo hizo referencia a algún tópico. De estos dos tercios, a más de la mitad $(58 \%)$ le hubiese gustado recibir información sobre los "pasos a seguir", a un $18 \%$ información sobre las "distintas causas de la infertilidad" y a un $24 \%$ ser informada acerca de las "reales posibilidades de éxito y/o fracaso del tratamiento".

Con respecto a la información que le hubiese gustado recibir DURANTE, del total de mujeres que mencionó algo en este aspecto ( 35 casos), una proporción considerable dijo que esperaba recibir información sobre su "caso personal". Prácticamente seis de cada diez mujeres esperaba que el especialista le diera una explicación exhaustiva sobre los factores que estaban limitando el funcionamiento de su aparato reproductor. Una quinta parte hizo mención al deseo de recibir información sobre sus "posibilidades reales de embarazo". Una proporción menor (14\%) esperaba que el médico le diera explicaciones de los exámenes que él le solicitaba. Finalmente un $6 \%$ dijo que le hubiese gustado saber acerca de la "duración" del tratamiento.

\section{Opiniones de la mujer infértil sobre el tratamiento}

Los datos revelan que a la mayoría $(60 \%)$ de las mujeres les fue desagradable la "demora en la atención", un 18\% declaró que "no hubo demora" y un 16\% que ésta

* Las seis restantes no tenían conocimientos al respecto. 
fue "agradable" en tanto les sirvió para conversar con otras mujeres e informarse acerca de los aspectos del mismo tratamiento.

Más de los dos tercios de los casos que estuvieron más de tres meses en tratamiento (41 casos) encontró "desagradable" la lentitud del tratamiento. No sólo sintieron que se prolongaba demasiado, sino también más de la mitad $(56 \%)$ sintió que no tenía sentido continuar con éste. Un porcentaje menor $(19 \%)$ dijo que era "normal" que se prolongara y sólo un 10\% consideró "agradable" esta situación.

Interesó conocer también la opinión de la mujer en relación con la comunicación que tuvo con el especialista; de aquellas que dijeron que el médico fue "poco comunicativo" (29 casos), más de dos tercios opinó que esta situación fue "muy desagradable". Del grupo restante, un $17 \%$ dijo que "a veces" fue poco comunicativo y que esto era "agradable" y sólo un 10\% respondió que estaba conforme con que el médico fuera "poco comunicativo".

La mayoría $(64 \%)$ de las mujeres no está conforme con que los resultados de los exámenes no permitan hacer un diagnóstico inmediato y definitivo. Del grupo restante (18 casos), menos de un tercio manifestó que los resultados fueron a corto plazo. Un $10 \%$ opinó que era "normal y lógica" la demora en el diagnóstico.

Las tres cuartas partes de las mujeres declararon que durante las consultas había existido "mucha privacidad", menos de un cuarto $(20 \%)$ dijo que sólo existió "algo de privacidad" y un 10\% que "no hubo privacidad". Las mujeres que dijeron sentirse cómodas con "mucha y alguna privacidad" se refirieron al hecho que aquélla permitía conversar tranquilamente con el especialista sobre su caso personal y aquél se encontraba exclusivamente dedicado a ellas.

Un análisis de las opiniones que tiene la mujer sobre los posibles daños que produce el tratamiento revela que es mayor el porcentaje de mujeres que cree que el tratamiento le hace daño a la mujer $(34 \%)$ que al hombre $(18 \%)$. Casi dos tercios de las mujeres que opinaron que el tratamiento es dañino para la mujer hicieron referencia a "daños en el sistema nervioso" (ansiedad, mal humor, irritabilidad), menos de un tercio dijo que el daño era "físico". El porcentaje restante $(12 \%)$ aludió a "daño moral" y que era "ni bueno ni malo", es decir manifestó una opinión neutral.

Con respecto al daño que podría causarle al esposo, nueve de cada diez mujeres aludieron a "daño psicológico": sentimiento del ridículo por ser objeto de bromas y frustración. Sólo una de cada diez mujeres dijo que le producía "daño físico". La mayoría de las entrevistadas coincidieron en que "después de los 35 años" es la edad más arriesgada para que la mujer se embarace. Un $4 \%$ dijo que "entre los 30 y 34 años" y sólo un $6 \%$ declaró que no habría una edad más arriesgada. 
Transtornos fisiológicos asociados a estados emocionales y cambios en el estado afectivo aparecidos después de iniciado el estudio de la infertilidad

Se les preguntó a las mujeres si percibieron en ellas algunos cambios tanto fisiológicos como psicológicos después de iniciado el estudio de su infertilidad. Una clasificación de las respuestas indica lo siguiente:

$\begin{array}{ll}\text { Transtornos del sueño } & 27 \% \\ \text { Disminución del apetito } & 15 \% \\ \text { Tendencia al llanto } & 40 \% \\ \text { Irritabilidad y mal humor } & 54 \% \\ \text { Ansiedad } & 88 \%\end{array}$

Con respecto a los cambios en el estado afectivo, los datos revelan la presencia de sentimientos y emociones más bien negativos. Al ordenar, según la cantidad de mujeres que las mencionó, se obtuvo lo siguiente:

$\begin{array}{ll}\text { Ira } & 63 \% \\ \text { Desesperanza } & 59 \% \\ \text { Frustración } & 58 \% \\ \text { Pesimismo acerca de la posibilidad de embarazo } & 53 \% \\ \text { Baja autoestima en relación al rol femenino } & 51 \% \\ \text { Culpabilidad por no lograr un embarazo } & 35 \% \\ \text { Rechazo frente a mujeres embarazadas } & 33 \%\end{array}$

En relación con los temores, más de la mitad del grupo (51\%) sintió temor "a los exámenes". Más de un tercio (36\%) temor a "esterilidad definitiva" y un $15 \%$ a "la pérdida del esposo".

Relación de pareja y tratamiento de la infertilidad

Al preguntárseles a las mujeres cuál era el comentario que su esposo le hacía cuando ella iba a consulta, hallamos que nueve de cada diez esposos manifestaron un comentario favorable: le deseaba que "le fuera bien", querian ser informados de lo que habia ocurrido durante la consulta, preguntaban si su presencia era requerida y aconsejaban a su esposa sobre lo que debía conversar con el médico. De las que declararon que el esposo presentó una actitud desfavorable, aludieron que ésta estaba asociada al "costo", a la "inutilidad del tratamiento" e incluso algunos insultaban a su mujer ya que estaban en desacuerdo en continuar con el estudio.

Sólo un $14 \%$ le dijo a su esposa que dejara el tratamiento, un $36 \%$ hizo comenta- 
rios sobre "el costo" y un $42 \%$, dado que no se lograba un embarazo le recomendó a su mujer que "buscara nuevas opiniones".

Al considerar las reacciones del esposo cuando tuvo que hacerse algún examen se constató que, contrariamente a lo que puede esperarse los sujetos más educados presentan una disposición menos favorable.

Cuadro 1

DISPOSICIÓN PARA HACERSE LOS EXÁMENES

Y NIVEL EDUCACIONAL

\begin{tabular}{lcccc} 
& \multicolumn{2}{c}{ Educación } & & \\
Disposición & Básica & Media & Superior & Total \\
\hline \multirow{2}{*}{ Favorable } & $\%$ & $\%$ & $\%$ & $\%$ \\
Desfavorable & 50 & 62 & 36 & 48 \\
\hline & 50 & 38 & 64 & 52 \\
\hline & 100 & 100 & 100 & \\
& $(10)$ & $(16)$ & $(22)$ & $(48)^{*}$
\end{tabular}

En los extremos de la variable Educación (BAJO y ALTO) se encuentra el mayor porcentaje de individuos desfavorablemente dispuestos a los exámenes e incluso, entre los de Nivel Educacional Alto es mayor la proporción con disposición desfavorable que aquellos en similar categoría entre los individuos de Nivel Educacional Básico. En cambio sólo un poco más de un tercio de los individuos de Nivel Medio reaccionan negativamente a los exámenes y en este grupo se encuentra la mayor proporción de individuos con disposición favorable $(62 \%)$.

Se les preguntó a las mujeres si, según su opinión, la relación con su pareja experimentó algún cambio después de iniciado el estudio de la infertilidad. Las respuestas indican que en una proporción importante $(44 \%)$ la relación con su esposo se tornó "más afectiva", siendo menor la proporción $(38 \%)$ de aquellas que declararon que la relación se volvió "tirante y distante". Un $12 \%$ dijo que su esposo manifestó "preocupación por los remedios" que ella debía ingerir (hora de ingestión, cantidad, etc.) y un $6 \%$ dijo que las relaciones sexuales carecieron de naturalidad.

También se les preguntó a las mujeres si se produjo algún cambio "después" de dejar de asistir a tratamiento. Del total de mujeres entrevistadas, un tercio respondió afirmativamente. En una proporción considerable de este grupo el cambio se manifestó en términos de "tranquilidad y armonía". Prácticamente seis

* A dos esposos no les fue solicitado el espermiograma. 
de cada diez mujeres se refirió a este cambio. Los cambios desfavorables o negativos fueron menos mencionados; un $25 \%$ declaró que se produjo un "distanciamiento" y en un $14 \%$ le "recriminó haber dejado el tratamiento".

Los datos dan cuenta de que el hombre participa en el estudio aun cuando, por razones de trabajo, no siempre puede acompañar a su mujer a la consulta. Al comparar los Estratos Socioeconómicos, es en el Estrato Bajo donde la asistencia es menor y estas mujeres declararon que les gustaría que el médico socilicitara la asistencia del esposo.

Con respecto a la opinión del esposo cuando la mujer decidió dejar de asistir al estudio de su infertilidad, un poco más de las tres cuartas partes de los esposos estuvieron de acuerdo con la decisión. Un 10\% no dio opinión, sólo un $8 \%$ se molestó ya que deseaba continuar en tratamiento y en un $4 \%$ la decisión fue iniciativa del esposo. Del total de mujeres, casi la cuarta parte $(24 \%)$ tomó la decisión por sí sola.

\section{Influencia social en relación al tratamiento}

En cuanto a las acciones realizadas con respecto al estudio de la infertilidad, la mayoría de las mujeres toman en cuenta en primer lugar la opinión de sus esposos $(56 \%)$, otras la del médico tratante $(24 \%)$ y en menor porcentaje $(20 \%)$ la de parientes y amigos.

El análisis de las actitudes hacia el tratamiento al ser clasificadas en Favorables, Neutral y Desfavorable, revela que los padres constituyen las personas más favorablemente involucradas; existe en la categoría Padres una proporción mayor $(68 \%)$ de casos que tuvieron una actitud favorable, que la de Parientes $(56 \%)$ y Amigos $(46 \%)$. La importancia que los padres le otorgan a este estudio se evidencia también en el hecho que ellos también son el grupo que presenta una mayor proporción de casos en actitud "neutral" ( $28 \%)$. Más de dos quintas partes de los amigos manifiestan esta actitud y más de un tercio en "parientes".

Los amigos constituyen el grupo que presentó una proporción mayor de casos con actitud "desfavorable" $(10 \%)$, seguido por los padres $(8 \%)$ y finalmente por parientes $(6 \%)$.

\section{Motivos de abandono del estudio de la infertilidad}

La mitad de las mujeres (50\%) aludió a motivos que hemos agrupados en la categoría "relación médico-paciente" (pérdida de confianza en el especialista, poca comunicación con el médico, rutinización y mecanización del tratamiento, trato dado a la paciente), una quinta parte se refirió a los "efectos del tratamiento (tensión, ansiedad, temor a exámenes), similar proporción $(20 \%)$ a motivos "económicos" y un 10\% a motivos de la "relación de pareja" (probable separación, poca cooperación de la pareja). 


\section{Estrato Socioeconómico y Motivos de Abandono}

Los datos indican que, independientemente del Estrato Socioeconómico, los motivos que prevalecen son aquellos agrupados en la categoría "relación médico-paciente". Sin embargo, es en el Estrato Socioeconómico Medio donde estos motivos están relativamente acentuados; ocho de cada diez mujeres aludieron a motivos que se refieren a este aspecto. Una proporción menor que constituye casi la mitad $(47 \%)$ de mujeres del Estrato Alto hicieron referencia a similares motivos y un $42 \%$ declaró lo mismo en el Estrato Socioeconómico Bajo. Los "efectos del tratamiento" conforman el segundo motivo mencionado entre mujeres de Estrato Alto $(33 \%)$ y Estrato Medio (12\%). En cambio en el Estrato Bajo el motivo que ocupa el segundo lugar es el "económico" $(31 \%)$.

Contrariamente a lo que se pueda espera las mujeres de Estrato Alto mencionaron el motivo "económico" $(13 \%)$, no habiendo ningún caso en esta categoría en mujeres de Estrato Medio. Los motivos de la categoría "relación de pareja" no fueron declarados por las mujeres de Estrato Medio, sí en cambio por las de Estrato Alto $(7 \%)$ y un poco más del doble es el porcentaje entre mujeres de Estrato Bajo $(15 \%)$.

Cuadro 2

ESTRATO SOCIOECONÓMICO Y MOTIVOS DE ABANDONO DEL TRATAMIENTO DE LA INFERTILIDAD

\begin{tabular}{lcccc}
\hline \multirow{2}{*}{ Motivos de Abandono } & \multicolumn{4}{c}{ Estrato Socioeconómico } \\
\hline & BAJO & MED & ALTO & Total \\
Relación Médico-Paciente & $\%$ & $\%$ & $\%$ & $\%$ \\
Relación de Pareja & 15 & 78 & 47 & 50 \\
Efectos del Tratamiento & 12 & 0 & 7 & 20 \\
Económicos & 31 & 0 & 33 & 20 \\
\hline & 100 & 100 & 100 & 100 \\
& $(26)$ & $(9)$ & $(15)$ & $(50)$
\end{tabular}

\section{DISCUSIÓN}

La literatura sobre infertilidad ha demostrado consistentemente que la pareja enfrenta una crisis debido a esta situación tan inesperada. Los datos obtenidos a través de este estudio exploratorio son concordantes con estos planteamientos. $\mathrm{Ha}$ quedado en evidencia que la mujer que está en tratamiento tanto por las demandas que éste supone como por el proceso mismo de crisis que experimenta, se encuentra afectada emocionalmente e incluso como fue declarado por ellas, estos mismos disturbios (ansiedad, irritabilidad, mal humor, etc.) en combinación con otros 
factores o por sí solos las lleva a tomar la decisión de discontinuar o abandonar el tratamiento. Las presiones externas emanan, en parte, del tratamiento: hacerse exámenes, tomar medicamentos, pedir permiso en el trabajo para asistir a consulta o examen, dar explicación por no quedar embarazada, tener relaciones sexuales con el esposo cuando el médico lo sugiere, etc. A este conjunto de demandas externas se agregan las internas: la vivencia de la crisis de infertilidad que la hace experimentar un conjunto de emociones negativas; frustración, ira hacia sí misma por ser incapaz de concebir generándole sentimiento de culpa, desesperanza y por ende una falta de sentido de su vida, pesimismo sobre la posibilidad de éxito del tratamiento. Debemos destacar que más de la mitad del grupo estudiado declaró sentirse "incompleta como mujer".

Varios estudios han comprobado que la intervención de los médicos y personal paramédico asumiendo la tarea de consejo y apoyo a la pareja en esta crisis, contribuye favorablemente a su resolución $(4,7,15,19,20,21)$. Al respecto los datos recogidos estarian indicando que la mujer recibe escaso o ningún apoyo emocional para enfrentar esta situación tan inesperada; la alta frecuencia de mujeres que reconocieron que estaban irritable $(54 \%)$ y "ansiosas" $(88 \%)$ durante el tratamiento, junto con la alta frecuencia de casos en la categoria "motivos de la relación médico-paciente" nos lleva a pensar que así ocurrió.

Esta carencia de soporte emocional podría estar agudizando la falta de bienestar psicológico que se sabe afecta a la mujer infértil (5) y contribuiría a aumentar el riesgo de abandono y discontinuación del tratamiento.

La importancia del soporte emocional también fue destacado por Ben Sira (2) quien comprobó que aquél es un factor determinante en la evaluación del paciente de la eficacia del tratamiento como de la satisfacción con éste. Otro estudio realizado en nuestro medio (18) planteó conclusiones similares: existe una asociación entre la Calidad de la Comunicación Médico-Paciente y la Opinión de la Atención Recibida. En otras palabras, mientras mejor sea la comunicación con el médico, el paciente tendrá una evaluación más favorable del tratamiento, trato personal e interés que el médico le manifieste y más confianza depositará el paciente en el especialista.

\section{CONCLUSIONES}

Un agente de salud fue el medio de contacto más frecuente entre la mujer y el médico especialista.

La mujer inicia el estudio de la infertilidad con un alto grado de motivación lo que quedó demostrado en la cantidad de mujeres que, a pesar de haber tenido motivos para dejar de asistir a tratamiento, se encuentra nuevamente en estudio. Además más de un tercio del grupo estuvo en estudio con otro u otros especialistas. 
En general la mujer cuando está en estudio y no ha decidido aún abandonarlo o cambiar de especialista mantiene su asistencia regularmente.

Las expectativas acerca del tipo de exámenes, costo y duración del tratamiento son escasamente coincidentes con la realidad que enfrenta la mujer infértil. En este sentido es dable suponer que el bajo cumplimiento de las expectativas estaría afectando el nivel de satisfacción con el tratamiento y probablemente contribuyendo a la decisión de abandono de aquél.

El bajo nivel de conocimientos que tiene la mujer antes de iniciar el estudio explicaría, en parte, las expectativas poco realistas que elabora.

El bajo nivel de tolerancia a los exámenes expresa el compromiso emocional de la paciente con su infertilidad; la ansiedad y los temores son debido al desconocimiento de los exámenes como la posibilidad de que los resultados permitan establecer el diagnóstico de esterilidad definitiva.

Los problemas que enfrenta la mujer para la realización de los exámenes van condicionando, en alguna medida, su disposición a continuar o a abandonar el tratamiento. Se pudo constatar que el problema más recurrente, entre mujeres que trabajan, es tener que pedir permiso y faltar al trabajo para hacerse algún examen. El dinero y las discusiones con la pareja fueron los problemas mencionados en segundo lugar. Probablemente las discusiones con la pareja se presentan en el grupo que está conformado por aquellos esposos que les disgustó o simplemente no estuvieron dispuestos a hacerse el espermiograma. La negativa del esposo por participar en la realización de los exámenes puede deberse a muchas razones: temor a que los exámenes revelen que ellos son los infértiles y no la mujer, el sentimiento de pérdida de virilidad al masturbarse o la ausencia de discreción de parte de algunas personas que trabajan en los laboratorios que, sin quererlo, hacen evidente el procedimiento de extracción de muestra de semen.

La mujer está informada sobre los posibles factores femeninos y masculinos que impiden se produzca el embarazo. Sin embargo sus conocimientos son escasos en relación al estudio y tratamiento de la infertilidad; una proporción alta de mujeres inicia el estudio desconociendo los pasos por seguir y de aquellas que tienen algún conocimiento, éste es general (posibles causas, es "prolongado", "efectivo", "dolorosos" y "se estudia a la mujer").

La mujer desea ser informada antes y durante el estudio y tratamiento de su infertilidad. Antes de iniciar el estudio le hubiese gustado ser informada de las acciones por seguir indicadas por el especialista para el manejo de su infertilidad y esperaba que durante el estudio el médico se explayara informándole acerca de su problema de infertilidad.

Las mujeres sustentan una actitud más bien negativa hacia algunos aspectos del estudio y tratamiento de la infertilidad. Esta actitud quedó en evidencia a través de las opiniones desfavorables que dieron acerca de la demora en la atención, duración del tratamiento, médico poco comunicativo y resultados de los exámenes 
poco concluyentes. Si consideramos que la calidad de la comunicación en la relación médico-paciente influye en la evaluación del paciente acerca del tratamiento y del médico, es dable esperar que particularmente aquellas mujeres que fueron atendidas por especialistas poco comunicativos, constituyen un grupo de alto riesgo de abandono, dado que no están satisfechas con la atención recibida.

Las mujeres estudiadas presentan un conjunto de emociones y sentimientos negativos: ira, desesperanza, frustración, pesimismo, culpabilidad, rechazo al ver una mujer embarazada, baja autoestima en su rol de mujer. Lo ocurrido en estas mujeres confirma la existencia de una crisis de infertilidad la cual ha sido ya expuesta por estudiosos del tema $(4,5,12,13,15,19,21)$.

El impacto y compromiso que supone la situación de infertilidad como su estudio y tratamiento no sólo se reflejan en lo afectivo sino también en lo fisiológico; las mujeres reconocieron que experimentaron ansiedad, trastornos del sueño, tendencia al llanto, irritabilidad y mal humor. Difícil es establecer una línea demarcatoria que permita separar la influencia de factores culturales (valores, creencias), sociales (presión y estigma social, expectativas de rol no satisfechas) y psicológi$\cos$ (motivación, afectos) en el impacto de la infertilidad en la mujer.

Cualquiera sea el o los factores que hacen que la mujer esté afectiva y fisiológicamente alterada, estamos convencidos de que, toda vez que el estudio y tratamiento es prolongado, el médico especialista debería complementar su desempeño profesional con algún tipo de conducta afectiva de manera de disminuir la ansiedad generada tanto por la infertilidad, como por su estudio y tratamiento. Aun cuando no se ha logrado establecer con certeza en qué medida y cómo lo afectivo influye en la capacidad reproductiva, creemos que el manejo de este componente es una condición necesaria que aumenta las probabilidades de éxito en el tratamiento, disminuye el riesgo de abandono y contribuye a una resolución favorable de la crisis de infertilidad cuando no es posible lograr la deseada concepción.

AGRADECIMIENTOS

Este articulo es parte del Proyecto S-2655-8712 realizado por la autora y el profesor Licer Viveros C., Director del Departamento de Sociologia, financiado por el Departamento Técnico de Investigación (D.T.I.), Universidad de Chile.

La autora desea expresar sus agradecimientos a todos los médicos especialistas en infertilidad quienes aportaron información y sugerencias en torno al problema y otorgaron facilidades para el acceso a sus Consultas y Servicios Públicos de 


\section{BIBLIOGRAFÍA}

1. Abse, D.W. Psychiatric aspects of human male infertility. Fertility and Sterility. Vol. 17: 133-139, 1966.

2. BEN-SIRA, Z. Affective and instrumental components in the Physician-patient relationship: and additional dimension of interaction theory. Journal of Health and Social Behavior. Vol. 21: 170-180, 1980.

3. Bos, C.; Cleghorn, R.A. Psychogenic Sterility. Fertility and Sterility. Vol. 9: 84-95, 1958.

4. Bresnick, E.; TAYMor, M. The role of Couseling in infertility. Fertility and Sterility. Vol. 32: 154-156, 1979 .

5. CaLlan, V.J. The personal and marital adjustment of mothers and of voluntarily and involuntarily childless wives. Journal of Marriage and the family. Vol. 49: 847-856, 1987.

Perceptions of parenthood and childlessness: a comparison of mothers and voluntarily childiess wives. Population and Environment. Vol. 6: 179-189, 1983.

6. Ford, E.S.C. et al. A psychodynamic approach to the study of infertility. Fertility and Sterility. Vol. 4, 456-465, 1953.

7. Guerrero, C.D. Management of the infertile Couple: 25 years' experience. Fertility and Sterility. Vol. 15: 534-541, 1964

8. Jehoshua, D.; Homburg, R.; Rabau, E.. An evaluation of etiologic factors and therapy in 665 infertile couples. Fertility and Sterility. Vol. 28: 718-722, 1977

9. Karow, A.M. Letters to the editor. Fertility and Sterility. Vol. 38: 392-393, 1982.

10. LAMB, E.J. Prognosis for the infertile couple. Fertility and Sterility. Vol. 23: 320-325, 1972.

11. MACLEOd, A.W. Some psychogenic aspects of infertility. Fertility and Sterility. Vol. 15: 124-134, 1964.

12. Mahlstedt, P.P. The psychological component of infertility. Fertility and Sterility. Vol. 43: 335-346, 1985.

13. Matthews, A.M.; Matthews, R. Beyond the mechanics of infertility: perspectives on the social psychology of infertility and involuntary childlessness. Family Relations. Vol. 35: 479-487, 1986.

Infertility and Involuntary childlessness: the transition to nonparenthood. Journa/ of Marriage and the Family. Vol. 48: 641-649, 1986.

14. Marshall, J.R. Infertilidad en Diagnóstico y tratamiento Ginecoobstétricos de Ralph Benson. Edit. El Manual Moderno, México, pp. 919-935, 1982.

15. Menning, B.E. The emotional needs of infertile couples. Fertility and Sterility. Vol. 34: 313-319, 1980.

Resolve: a support group for infertile couples. American Journal of Nursing. Vol. 76: 258-259, 1976.

16. MIALL, $C_{H} . E$. Perceptions of informal sanctioning and the stigma of involuntary childlessness. Deviant Behavior. Vol. 6: 383. 403, 1985.

17. Noyes, R.W.; ChapNick, E.M. Literature on psychology and infertility. Fertility and Sterility. Vol. 15: 543-558, 1964.

18. Pincheira, A.; Fuenzalida, M.S. La comunicación médico-paciente en su relación con la atención médica. Un análisis sociológico en el Sisterna Estatal y Privado. Tesis para op. tar al grado de Licenciada en Sociología. Universidad de Chile, 1983. 
19. Porter, N.L.; Scott, C. Infertility: towards an awareness of need among family life practitioners. Family Relations. Vol. 33: 309-315, 1984.

20. Rosenfeld, D.L.; Mitchell, E. Treating the emotional aspects of infertility: counseling services in an infertility clinic. Am. J. Obstet.
Gynecol. Vol. 17: 177-179, 1979.

21. Seibel, M.M.; Taymor, M.L. Emotional aspects of infertility. Fertility and Sterility. Vol. 37: 137-145, 1982.

22. WALKER, H.E. Psychiatric aspects of infertility. The Urologic Clinics of North America. Vol. 5: 481-488, 1978.

Dirección del autor:

Cristina Di Silvestre. Socióloga. U. de Chile.

Departamento de Sociologia. Diagonal Paraguay $265,4^{\circ}$ piso.

Universidad de Chile. Teléfono: 222.95.12-233 